Recepción: 12 / 02 / 2018

Aceptación: 28 / 04 / 2018

Publicación: 05 / 06 / 2018

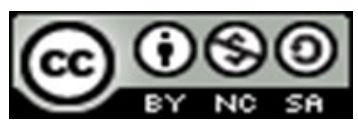

Ciencias de la salud

Artículo de Investigacion

\title{
Daño cerebral adquirido. Acercamiento
}

gnoseológico Acquired brain damage. Gnoseological

approach Dano cerebral adquirido. Abordagem

\author{
gnoseological
}

\author{
Clelia C. Ricaurte-Jijón ${ }^{\mathrm{I}}$ \\ clelia.ricaurte@uleam.edu.ec \\ Angie M. Anton-Cedeño ${ }^{\mathrm{II}}$ \\ anguieanton@hotmail.com
}

Correspondencia: clelia.ricaurte@uleam.edu.ec

\footnotetext{
I Especialista en Pediatría, Diploma Superior en Desarrollo Local y Salud, Doctora en Medicina y Cirugía, Docente en la Universidad Laica "Eloy Alfaro" de Manabí, Manta, Ecuador.

II Licenciada en Terapia Ocupacional, Docente en la Universidad Laica "Eloy Alfaro" de Manabí, Manta, Ecuador.
} 


\title{
Resumen
}

El daño cerebral adquirido está aumentando progresivamente por la conjunción de dos frentes: por un lado, la mayor edad de la población y, por otro, el aumento de la supervivencia de todos los procesos neurológicos graves, debido a la mejora en los servicios regionales de emergencia y los avances en los medios diagnósticos y terapéuticos. A tales efectos, se realizó una revisión bibliográfica exhaustiva para exponer algunos aspectos relacionados con esta afección (definición, factores de riesgo, aspectos vitales para el diagnóstico, secuelas, rehabilitación, cuadro clínico, entre otros). Un mejor conocimiento de esta patología, permitirá la evolución y calidad de vida de los pacientes y familiares.

Palabras clave: Lesión cerebral; accidente cerebrovascular; traumatismo craneoencefálico; rehabilitación.

\begin{abstract}
Acquired brain damage is progressively increasing due to the combination of two fronts: on the one hand, the greater age of the population and, on the other, the increase in the survival of all the serious neurological processes, due to the improvement in the regional services of emergency and advances in diagnostic and therapeutic means. To this end, an exhaustive bibliographic review was carried out to expose some aspects related to this condition (definition, risk factors, vital aspects for the diagnosis, sequelae, rehabilitation, clinical picture, among others). A better knowledge of this pathology will allow the evolution and quality of life of patients and relatives.
\end{abstract}

Keywords: Brain injury; cerebrovascular accident cranioencephalic trauma; rehabilitation.

\section{Resumo}

O dano encefálico adquirido está progressivamente aumentando devido à combinação de duas frentes: por um lado, a maior idade da população e, por outro, o aumento da sobrevivência de todos os processos neurológicos graves, devido à melhora nos serviços regionais de saúde. emergência e avanços nos meios diagnósticos e terapêuticos. Para tanto, foi realizada uma revisão bibliográfica exaustiva para expor alguns aspectos relacionados a essa condição (definição, fatores de risco, aspectos vitais para o diagnóstico, sequelas, reabilitação, quadro clínico, entre outros). Um melhor conhecimento desta patologia permitirá a evolução e qualidade de vida de pacientes e familiares. 
Palavras chave: Lesão cerebral; acidente vascular cerebral traumatismo cranioencefálico; reabilitação.

\section{Introducción}

El daño cerebral adquirido (DCA), es una problemática de salud de alto impacto a nivel mundial y de especial relevancia en países industrializados, tanto por su alta incidencia y su predominio en la población joven. Además del elevado número de muerte que ocasiona, cabe destacar los casos de discapacidad derivados de estas lesiones. ${ }^{1}$

La incidencia del daño cerebral adquirido, se produce en una franja de edad que suele ir desde los 16 a 35 años aproximadamente y es más frecuente en hombres que en mujeres. Aunque esta sea la incidencia, tenemos que destacar que cada DCA es singular, no tiene edad, ni sexo, ni situación socio - cultural, cualquier persona puede padecerlo. Nadie está exento de ello. ${ }^{2}$

Se estima que unos 57 millones de personas han sido hospitalizadas en el mundo, debido a un traumatismo craneoencefálico, pero el número de personas que viven con las graves secuelas se desconocen.$^{3}$

Según se ha citado, la Federación Española de Daño Cerebral (FEDACE) el 27 de octubre del 2017 planteo que cada año 104.000 personas sufren daño cerebral. ${ }^{4}$

\section{Desarrollo}

\section{Definición}

El término daño cerebral adquirido hace referencia a una lesión en el cerebro que se produce con posterioridad al momento del nacimiento. Las causas del daño cerebral adquirido son múltiples y su origen puede ser traumático (accidentes de tráfico, caídas, golpes, etc.) o no traumático (accidentes vasculares, tumores cerebrales, infecciones, hipoxia o isquemia, intoxicaciones, etc.). A pesar de la amplitud de patologías que incluyen, la mayoría de las definiciones de DCA no consideran como tal a los trastornos degenerativos.

Hay que destacar también que no afectará del mismo modo a un cerebro en desarrollo (niños), que a un cerebro completamente desarrollado (adultos). ${ }^{5}$ 
En este orden de ideas, el daño cerebral adquirido engloba daños cognitivos, motores conductuales y físicos que afectan y comprometen la vida del individuo.

\section{Factores de riesgo y causas del daño cerebral adquirido}

Entre los factores de riesgo más sobresalientes que pueden ocasionar un DCA, podemos destacar: el consumo excesivo de alcohol, el abuso de drogas, la conducción irresponsable y temeraria, la falta de protección obligatoria entre otras.

El daño cerebral adquirido (DCA) puede originarse por diferentes causas: exógenas como traumatismo craneoencefálico, encefalopatía por tóxico (fármacos, drogas de abuso o sustancias químicas), encefalopatía por agentes físicos (radiación ionizantes, hipertemia, hipotermia), enfermedades infecciosas; y las causas endógenas como ictus isquémicos o hemorrágicos, encefalopatía anóxica, neoplasias primarias o secundarias, enfermedades inflamatorias autoinmune, enfermedades del tejido conectivo, vasculitis, enfermedades desmienilizantes. ${ }^{6}$

\section{Las fases por los que pasa la persona tras sufrir el DCA son:}

$\checkmark$ Fase Crítica: La persona se encuentra hemodinámicamente estable, pero persiste la probabilidad de aparición de complicaciones, existiendo aún riesgo para su vida. Esta fase se desarrolla en las Unidades de Cuidados Intensivos de los Hospitales y/o en las plantas de neurología/neurocirugía.

Fase Aguda: La persona se encuentra estable neurológicamente, disminuyendo el riesgo de presentar complicaciones. Comienzan a identificarse las secuelas a nivel físico, cognitivo, conductual y funcional. Se mantiene la atención en régimen hospitalario y la duración es independiente para cada persona. Generalmente, esta fase se desarrolla en las plantas de los Hospitales o en las unidades de ictus hospitalarias.

$\checkmark$ Fase Rehabilitadora: Tras el alta hospitalaria, comienza el periodo de rehabilitación especializada. Esta fase incluye la rehabilitación en régimen de hospitalización, así como el tratamiento de manera ambulatoria cuando la persona ya no requiere de asistencia médica continua. Si las secuelas se mantienen con carácter crónico, la atención se desarrollará en diferentes recursos sociosanitarios especializados, como son los Centros de Día, Pisos Tutelados, Atención Ambulatoria. ${ }^{7}$ 


\section{Sintomatología en función de la zona dañada:}

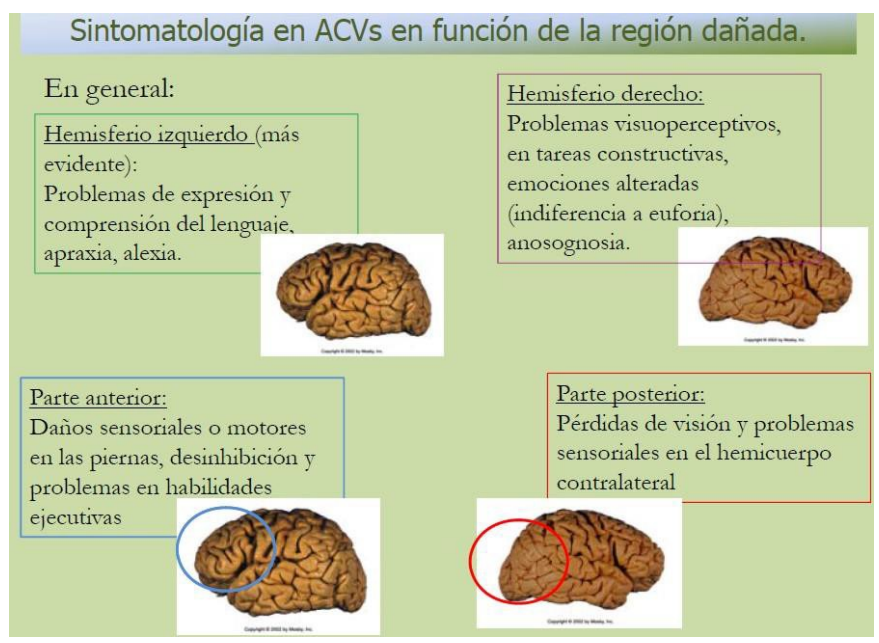

$\checkmark$ Hemisferio izquierdo: suelen ser más evidentes, porque se daña el lenguaje. Problemas de expresión y comprensión del lenguaje, apraxias, alexia (capacidad de leer). No hay problemas motores.

$\checkmark$ Hemisferios derechos: problemas visuoperceptivos, en tareas constructivas, emociones alteradas (indiferencia a euforia) y anosognosia (Tendencia a infravalorar el problema que se tiene).

$\checkmark$ Parte anterior: Daños sensoriales o motores en las piernas, deshinbición y problemas en habilidades ejecutivas.

$\checkmark$ Parte posterior: pérdidas de visión y problemas sensoriales en el hemicuerpo contralateral. $^{8}$

A manera de resumen los síntomas más comunes son: impulsividad, capacidad deficiente de planificación y de tomar decisiones, temas de conversación inapropiados, falta de motivación, dolor de cabeza persistente, confusión constante, lentitud al pensar o actuar, pérdida del equilibrio, visión borrosa, cambios en los patrones de dormir, zumbido en los oídos, vértigo, fatiga permanente, mala memoria. 


\section{Diagnóstico}

Por lo general, las lesiones cerebrales traumáticas constituyen emergencias, y las consecuencias pueden empeorar rápidamente si no se brinda tratamiento

\section{$\checkmark$ Escala de coma de Glasgow}

Esta prueba de 15 puntos le permite al médico, o a otros miembros del personal médico de urgencia, evaluar la gravedad inicial de una lesión cerebral al comprobar la capacidad de la persona de seguir instrucciones, y de mover los ojos y las extremidades. La coherencia del habla también brinda indicios importantes.

En la escala de coma de Glasgow, las capacidades se califican con un número que va de 3 a 15 . Las calificaciones altas significan lesiones menos graves.

\section{$\checkmark$ Pruebas de diagnóstico por imágenes}

Exploración por tomografía computarizada (TC). Esta suele ser la primera prueba que se realiza en la sala de urgencias cuando hay sospechas de una lesión cerebral traumática. En una exploración por tomografía computarizada, se usa una serie de radiografías para crear una vista detallada del cerebro. En una exploración por tomografía computarizada, rápidamente se pueden visualizar fracturas y descubrir evidencias de sangrado en el cerebro (hemorragia), coágulos sanguíneos (hematomas), tejido cerebral con hematomas (contusiones) e hinchazón del tejido cerebral.

Resonancia magnética (RM). Una RM usa poderosas ondas de radio e imanes para crear una vista detallada del cerebro. Esta prueba se puede usar una vez que el trastorno de la persona se haya estabilizado o si los síntomas no mejoran poco después de la lesión. ${ }^{9}$

El daño cerebral puede afectar a todas las áreas del funcionamiento del ser humano. Los déficits dependerán del tipo de lesión, la localización y severidad inicial de la misma... así como de características propias de cada afectado como la edad, personalidad o capacidades previas a la lesión. Las secuelas se agrupan en cuatro dimensiones que pueden solaparse o no en una misma persona: ${ }^{10}$ 
Déficits físico-motores: Pueden producirse alteraciones del equilibrio, incapacidad para la bipedestación (mantenerse de pie), incapacidad para la marcha, limitaciones en el movimiento de una o varias extremidades, o incluso el control del tronco y cabeza en situación de reposo. Otras alteraciones que pueden surgir son: temblores, falta de sensibilidad y movilidad fina en miembros del cuerpo, e incluso pérdida parcial o total de alguno de los sentidos como la vista.

Déficits Cognitivos: Podemos citar déficits de aprendizaje y memoria, déficits atencionales, alteraciones del lenguaje, alteraciones del pensamiento formal (razonamiento lógico-deductivo, resolución de problemas, abstracción) y alteraciones en la regulación de la conducta propositiva o dirigida a una meta (planificación, iniciación y autorregulación conductual). En definitiva, puede verse afectada la capacidad de pensamiento, toma de decisiones y control consciente de nuestra conducta.

$\checkmark$ Alteraciones de la comunicación: Estas secuelas se producen como consecuencia de la alteración de habilidades físicas y cognitivas o la combinación de las mismas. Cuando se trata de alteraciones físicas, se ve afectada la expresión oral (debido a la pérdida de control de los músculos fonoarticulatorios), escrita (pérdida de control de los miembros superiores) o de la comprensión (pérdida de la capacidad de audición o interpretación de sonidos).

Alteraciones Conductuales y/o Emocionales: A la par que las alteraciones cognitivas aparecen alteraciones conductuales y/o emocionales asociadas con cambios de carácter o personalidad. El origen de las alteraciones conductuales se podría considerar de dos formas: Falta de inhibición, lo que causa desinhibición conductual, en ocasiones la desinhibición provoca conductas física o verbalmente agresivas. Otras personas muestran exceso de inhibición o pobre iniciativa conductual; el afectado muestra apatía no iniciando actividades si no se le pide explícitamente, no disfruta tanto de las cosas que antes le gustaban, tiene sensación de agotamiento y letargo, y a nivel afectivo apenas expresa las emociones.

Con la experiencia del autor, no existe una persona con un daño cerebral igual a otro ya que puede tener unas secuelas diferentes a otra persona que ha pasado por semejantes circunstancia 
En el orden de las ideas anteriores, una característica frecuente en las personas con daño cerebral, es su falta de conciencia de los déficits derivados de la lesión cerebral (Anosognosia), especialmente cuando se trata de alteraciones cognitivas y emocionales. Si aparece este síntoma, frecuentemente la persona afectada rechaza la ayuda o dirección de quien le supervisa, dificultando su cuidado y rehabilitación.

Esta diversidad en las secuelas tras sufrir un DCA, hace imprescindible una evaluación individualizada previa al inicio de la rehabilitación. También es fundamental intervenir sobre su ambiente para que sea lo más rehabilitador y estimulante posible.

\section{Tratamiento}

El tratamiento depende de la gravedad de la lesión.

\section{La rehabilitación tras un daño cerebral}

El proceso rehabilitador comienza desde el momento de la aparición de la lesión. Pensar en daño cerebral es pensar en rehabilitación. La rehabilitación integra el conjunto de estrategias de intervención que tienen como objetivo reducir las alteraciones físicas, cognitivas, conductuales y funcionales que la persona tiene tras la lesión para minimizar el impacto en su vida cotidiana.

El término rehabilitación implica el trabajo hacia el restablecimiento de la situación de los pacientes al grado de funcionamiento más alto posible en el ámbito físico, psicológico y de adaptación social. Esto requiere poner todos los medios posibles para reducir el impacto de las condiciones que son discapacitantes, para permitir al paciente alcanzar su máximo nivel óptimo de integración social.

Existe un consenso unánime en que el inicio de la intervención debe ser precoz, para optimizar el proceso inicial de recuperación espontánea y aumentar la posibilidad de recuperación final. Asimismo, los estudios demuestran que debe ser un tratamiento intensivo (máxima intensidad que el paciente puede tolerar y que esté dispuesto a seguir) y prolongado (que la duración del tratamiento en el tiempo sea suficiente para ser efectivo).

El proceso rehabilitador debe garantizar la continuidad y coordinación asistencial. La rehabilitación es un continuo. Son diferentes etapas por las que pasa la persona y la familia, y se debe trabajar por garantizar un funcionamiento coordinado, desde los servicios sanitarios hasta la 
etapa de reinserción social. Existen varias fases por las que va a pasar la persona que ha sufrido la lesión y su entorno más próximo, cada una de estas fases tiene importantes objetivos.

- Fase crítica.

La mayoría de personas que sufren un daño cerebral son atendidas en servicios de urgencia, en unidades de cuidados intensivos, neurocirugía... Es una fase de importarte riesgo vital. El objetivo fundamental es la estabilización médica y prevención de complicaciones.

- Fase aguda.

El proceso de recuperación tras un traumatismo o un ictus, conlleva pérdida de conciencia seguido de un periodo de confusión, con un retorno gradual de las diversas funciones. Esta es la fase se identifica con el momento más próximo a la lesión.

- Fase subaguda o rehabilitadora.

Etapa de tratamiento intensivo donde la persona se encuentra estabilizada y es trasladada a la unidad de neurorehabilitación en régimen de hospitalización. Además de los cuidados médicos y enfermería, se inicia la rehabilitación intensiva.

Es importante destacar que las personas tras el daño cerebral tienen necesidades específicas en cada fase de recuperación. En determinados casos aparece deterioro funcional, por depresión, caídas, espasticidad, que puede requerir de la atención puntual del médico rehabilitador para volver al nivel de funcionamiento previo. En la fase crónica, aunque las secuelas se han estabilizado, puede ser necesario su apoyo para evaluar necesidades a largo plazo. ${ }^{11}$

El proceso rehabilitador requiere del equipo interdisciplicar una metodología de trabajo caracterizada por:

- Evaluación diagnóstica para conocer las necesidades asistenciales, de estado funcional y psicológico, nivel de dependencia y valoración del entorno familiar.

- Definición de objetivos a desarrollar en el plan terapéutico individual, en cada área, y realistas y relevantes para el paciente y la familia.

- Realizar las intervenciones y tratamientos necesarios. 
- Seguimiento y evaluaciones periódicas del plan terapéutico y los objetivos. Registro de los cambios que se produzcan.

- Reiniciar este ciclo del proceso rehabilitador, adaptándolo a la evolución de la persona y su entorno.

\section{$\checkmark$ Rehabilitación en el accidente cerebrovascular}

Hay una amplia evidencia sobre la efectividad de la rehabilitación multidisciplinaria en mejorar el estado funcional y el desempeño de las actividades de la vida diaria de los pacientes que han sufrido un ACV. Los efectos son mayores cuanto antes se inicie. Asimismo, el tratamiento de la fase aguda del ACV en unidades funcionales -unidades de ictus- mejora su supervivencia e independencia funcional al año.$^{12}$

\section{$\checkmark$ Rehabilitación en el traumatismo craneoencefálico}

Hay cierta evidencia en que el uso de ciertas estrategias de rehabilitación cognitivas y conductuales mejora la recuperación funcional en estos pacientes. En una revisión sistemática actualizada, se detecta que la mayoría de los pacientes con TCE leve se recuperan bien con información adecuada y sin intervención específica adicional; hay pruebas sólidas de que en los pacientes con daño cerebral severo-moderado, los programas rehabilitadores de gran intensidad conllevan recuperaciones funcionales más tempranas y más rápidas, así como reducciones en la duración de la rehabilitación. Sin embargo, hay una evidencia limitada de que con los programas realizados en el ambiente de la comunidad se puedan mejorar los grados de discapacidad. También hay acuerdo en la dificultad de estudiar las distintas actuaciones rehabilitadoras mediante las tecnologías tradicionales de investigación, en especial los ensayos clínicos aleatorizados, ya que en muchas ocasiones no sería ni ético ni útil. ${ }^{12}$

Después de lo anterior expuesto, las consecuencias del DCA, así como el consecuente proceso de neurorrehabilitación (NRHB), necesario para la máxima recuperación posible de las personas afectadas, es un proceso dilatado en el tiempo que puede extenderse incluso décadas. A lo largo de este proceso asistencial, intervienen diversos agentes en distintos ámbitos de atención y con objetivos complementarios que buscan como resultado final conseguir la máxima autonomía y participación social de la persona afectada y el restablecimiento de las redes sociales de las 
personas que no estando enfermas participan de los efectos de DCA (cuidadores, familiares, entorno laboral ...). La integración de todo el personal para el logro de este objetivo es hoy un reto. 13

Según Chaparro $\mathrm{M}^{14}$ en su investigación, concluyó que el aumento de la mortalidad del TCE grave, tiene causas multifactoriales, dentro de las que cabe mencionar la calidad de la atención medica pre-hospitalaria, las características de las lesiones primarias y el número creciente de pacientes con más de 60 años.

Así mismo, Ortega Sufiria et $\mathrm{al}^{15}$ en su estudio, el cual tuvo como objetivo describir el perfil clínico y principales factores pronóstico del traumatismo craneoencefálico leve. Resultados: El TCE leve fue más frecuentes en varones y el mecanismo causante más común fue el accidente de tráfico. El mayor por ciento de pacientes estuvo clasificado en bajo riesgo. Los enfermos con antecedentes médicos tienen una mayor probabilidad de desarrollar complicaciones intracraneales.

El análisis de las variables estudiadas y el estudio de investigaciones realizadas, reveló la elevada incidencia y prevalencia del DCA. Un mejor conocimiento de esta patología permitirá la evolución y calidad de vida de los pacientes y familiares.

\section{Referencias Bibliográficas}

1. Márquez Noriego B. Proceso de plasticidad cerebral en pacientes con daños adquirido. 2016. [citado 28 enero 2018]. Disponible en : https://idus.us.es/xmlui/bitstream/.../Blanca\%20Esther\%20Márquez\%20Noriego.pdf?

2. Día del daño cerebral adquirido. 26 de octubre de 2017 [citado 28 enero 2018]. Disponible en https://www.espaciologopedico.com/noticias/det/7751/dia-del-dano-cerebral-adquirido-26de-octubre-de-2017.html

3. Langlois $\mathrm{J}$ A. The Epidemilogy and impact of traumatic brain injury: a brief overview. J Head Trauma Rehabil 21 (5). 
4. Personas con daño cerebral adquirido y sus familiares 2017 [citado 28 enero 2018]. Disponible en: https://fedace.org/files/.../2017.../25-9-5345.admin.Manifiesto_17_lectura_facil_.pdf

5. Rios Lago M. Daño cerebral Adquirido: la necesidad de un trabajo multidiciplinar. Acción psicológica 2007; 4(3). Pp 5-7

6. Giménez Ates A. Guía de aproximación integral al daño cerebral adquirido. Fundación Casaverde. Alicante. 2015 [citado 15 diciembre 2017] Disponible en: https://www.grupocasaverde.com/.../manual-integral-del-dano-cerebral-adquirido.pdf

7. Palacios M, Ruíz M J, Tirado T, Dolz Llandres I, Asiain J R, Sánchez G. El daño cerebral adquirido, imprescindible actuar cuanto antes. 2016 [citado 8 enero 2018]. https://www.zonahospitalaria.com/el-dano-cerebral-adquirido-imprescindible-actuar-cuantoantes/

8. García Romero A. Daño cerebral: tipos, concepto, sintomatología. - Psicología y Conducta. 2016 [citado 8 enero 2018]. Disponible en: http://www.psicologiayconducta.com/danocerebral-tipos-sintomatologia.

9. Lesión cerebral traumatica . Diagnóstico y tratamiento [citado 8 enero 2018]. Disponible en. https://www.mayoclinic.org/es-es/diseases-conditions/traumatic-brain-injury/diagnosistreatment/drc-20378561

10. Daño cerebral adquirido. 2017 [citado 8 enero 2018]. Disponible en. http://neuroamune.com/dano-cerebral-adquirido/

11. Murga FM. Eficacia de la rehabilitación neurosicológica. 2006 [citado 8 enero 2018]. Disponible en: https://dialnet.unirioja.es/descarga/articulo/2262760.pdf

12. Alberdi Odriozola Fermín, Iriarte Ibarrarán Marta, Mendía Gorostidi Ángel, Murgialdai Arantza, Marco Garde Pilar. Pronóstico de las secuelas tras la lesión cerebral. Med. 2009 [citado 15 enero 2018]; 33(4): 171-181. Disponible en: http://scielo.isciii.es/scielo.php?script=sci_arttext\&pid=S0210-56912009000400004\&lng=es. 
13. Estrategia para la atención al daño cerebral adquirido en la comunidad de Valencia 20172020. Geralitad Valenciana. Consejería de Sanidad Universal. Salud pública 2009 [citado 15 enero 2018] Disponible en: www.san.gva.es/documents/156344/.../DCA_castellano_imprimible_dos_caras.pdf

14. Chaparro M W, Mosqueda Betancourt G, Varela Hernández A. Caracterización de los pacientes con traumatismo craneoencefálico graves atendidos en Camagüey. Rev Cubana Neurol-Neorocir. 2013; 3(1).

15. Ortega Sufiria J M, Lomillo P M, Choque C B, Tamarit D M, Poveda N P, López S M, López R A. Perfil clínico y principales factores pronóstico del traumatismo craneoencefálico leve. Rev Cubana Neurol-Neorocir. 2017; 7(1). 